\title{
Impaired calcineurin signaling in myeloid cells results in downregulation of pentraxin-3 and increased susceptibility to aspergillosis
}

\author{
T Zelante ${ }^{1,2}$, AYW Wong ${ }^{1,3}$, A Mencarelli $^{1,4}$, S Foo $^{1}$, F Zolezzi ${ }^{1}$, B Lee $^{1}$, M Poidinger ${ }^{1,5}$, \\ P Ricciardi-Castagnoli ${ }^{1}$ and $\mathrm{J}_{\text {Fric }}{ }^{1,6}$
}

Treatment of post-transplant patients with immunosuppressive drugs targeting the calcineurin-nuclear factor of activated T cells (NFAT) pathway, including cyclosporine A or tacrolimus, is commonly associated with a higher incidence of opportunistic infections, such as Aspergillus fumigatus, which can lead to severe life-threatening conditions. A component of the $A$. fumigatus cell wall, $\beta$-glucan, is recognized by dendritic cells (DCs) via the Dectin-1 receptor, triggering downstream signaling that leads to calcineurin-NFAT binding, NFAT translocation, and transcription of NFAT-regulated genes. Here, we address the question of whether calcineurin signaling in CD11c-expressing cells, such as DCs, has a specific role in the innate control of $A$. fumigatus. Impairment of calcineurin in CD11c-expressing cells $\left(\mathrm{CD} 11 \mathrm{c}^{\mathrm{cre}} \mathrm{Cnb1}{ }^{\text {loxP }}\right.$ ) significantly increased susceptibility to systemic $A$. fumigatus infection and to intranasal infection in irradiated mice undergoing bone marrow transplant. Global expression profiling of bone marrow-derived DCs identified calcineurin-regulated processes in the immune response to infection, including expression of pentraxin-3, an important antifungal defense protein. These results suggest that calcineurin inhibition directly impairs important immunoprotective functions of myeloid cells, as shown by the higher susceptibility of CD11c ${ }^{\text {cre }} \mathrm{cnb}^{\text {loxP }}$ mice in models of systemic and invasive pulmonary aspergillosis, including after allogeneic bone marrow transplantation. These findings are relevant to the clinical management of transplant patients with severe Aspergillus infections.

\section{INTRODUCTON}

It is well established that solid organ and hematopoietic cell transplant recipients have an increased susceptibility to various opportunistic infections. ${ }^{1}$ For instance, invasive aspergillosis (IA), a severe fungal infection associated with allogeneic hematopoietic stem cell transplantation, ${ }^{2,3}$ is a leading cause of mortality following hematopoietic cell transplantations ${ }^{4-6}$ and solid organ transplantations. ${ }^{7}$ Graft-versus-host disease (GvHD) and solid organ rejections are prevented using immunosuppressive drugs targeting calcineurin-nuclear factor of activated $\mathrm{T}$ cells (NFAT) signaling, such as cyclosporine A (CsA) and tacrolimus (FK506); however, both of these agents are linked with increased susceptibility to fungal infections. $^{3,8}$ This issue is of increasing concern with rising numbers of immunosuppressed patients developing uncontrolled fungal infections as a result of the recent spread of a number of strains resistant to conventional antifungal therapy. ${ }^{9}$

The calcineurin-NFAT pathway has been identified as an important player in the innate immune response ${ }^{10,11}$ with myeloid cells playing a key role in immune protection at the onset of IA infection. ${ }^{12}$ There is increasing evidence that inhibition of calcineurin-dependent signaling in innate myeloid cells has a severe impact on the control of various infections, ${ }^{10}$ specifically pertinent in antifungal protection. ${ }^{8,13}$ The molecular mechanism of increased susceptibility to fungal infections induced by inhibition of calcineurin in

${ }^{1}$ Singapore Immunology Network (SlgN), Agency for Science, Technology and Research (A*STAR), Biopolis, Singapore. ${ }^{2}$ Department of Experimental Medicine, University of Perugia, Perugia, Italy. ${ }^{3}$ National University of Singapore Graduate School for Integrative Sciences and Engineering, National University of Singapore, Singapore. ${ }^{4}$ Emerging Infectious Diseases Programme, Duke-NUS, Singapore. ${ }^{5}$ Department of Biological Sciences, National University of Singapore, Singapore and ${ }^{6}$ Center for Translational Medicine, International Clinical Research Center, St Anne's University Hospital Brno, Brno, Czech Republic. Correspondence: P Ricciardi-Castagnoli or J Fric, (paola.castagnoli@gmail.com or jan.fric@fnusa.cz) 
myeloid cells has not been fully explained. Given the severity and high mortality caused by fungal infections of immunosuppressed patients, the need for an improved understanding of the effect of calcineurin inhibitors on fungal immunity is clear.

Significant efforts have been made to prepare relevant models of fungal infection in calcineurin-deficient conditions, which revealed several new roles for calcineurin signaling in innate responses, including protection to Aspergillus fumigatus. ${ }^{14,15}$ More importantly, it has been shown that FK506 treatment in a model of transplant rejection therapy increased susceptibility to aspergillosis in a TLR9- and phagocytosisdependent manner. ${ }^{8,14}$ Earlier, Greenblatt et al. described an essential role for calcineurin in dendritic cells (DCs), macrophages, and neutrophils in protection against Candida. Mice treated with CsA or lacking calcineurin in lysozyme M (LysM)expressing cells were more susceptible to infection due to the impairment of a conserved fungal resistance pathway dependent on NFAT-regulated genes, such as interleukin-10 (IL-10) and cyclooxygenase-2. ${ }^{13}$ Impairment of calcineurin signaling has also been shown to be essential for the regulation of other TLR pathways and phagocytosis. ${ }^{14,16-18}$ Furthermore, calcineurin-NFAT plays an important role in myeloid progenitor maintenance as part of the Flt3 signaling cascade. ${ }^{17,19}$ The essential role of myeloid cells in IA protection has been confirmed by a significant mortality decrease when using non-myeloablating treatment strategies. ${ }^{7,20}$ The number of studies addressing the interaction between immunosuppressive drugs, myeloid cell function and the control of fungal infections remains limited, thus the potentially damaging effects of CsA and FK506 on the myeloid antifungal immune response may therefore be underestimated.

It is now well established that calcineurin signaling in DCs is triggered through TLR4-MD2 or Dectin-1. In DCs, the calcineurin-NFAT pathway is responsible for IL-2 secretion when cells are triggered with various pathogen-associated molecular patterns. ${ }^{21}$ We recently reported that IL-2 production by DCs is a key in the regulation of the Th17 response ${ }^{22}$ and in the development of intestinal inflammation. NFAT signaling is also important for the recognition of particulate forms of pathogens, ${ }^{14,18}$ highly relevant for protection against A. fumigatus spores. The main pathogen-associated molecular pattern-associated with recognition of fungi is $\beta$-glucan, which activates the NFAT pathway through Dectin-1 binding in DCs and macrophages. ${ }^{23}$

Pentraxin-3 (Ptx3) secretion is one of the major strategies used by myeloid cells to eliminate fungal infections. ${ }^{24,25} \mathrm{Ptx} 3$, a soluble secreted pattern recognition receptor, is induced in DCs, macrophages, and neutrophils upon TLR triggering ${ }^{26,27}$ and is linked to the phagocytic capacity of cells, ${ }^{28}$ as well as to the formation of neutrophil extracellular traps. ${ }^{29} \mathrm{Ptx} 3$ is able to recognize and bind A. fumigatus conidia, ${ }^{30}$ and functional impairment of Ptx 3 function is linked with a severe form of IA in patients following bone marrow $(\mathrm{BM})^{31}$ and solid organ transplants $^{32}$ as a result of lowered phagocytic activity and fungal clearance.
Here we address the role of calcineurin signaling in subsets of myeloid cells in mice with aspergillosis. Commonly used systemic treatments with CsA or FK506 do not allow the specific roles of myeloid cells to be properly dissected. We therefore designed a strategy to address the role of calcineurin in the immune response to A. fumigatus specifically in CD11cexpressing cells, using cre/loxP mice that lack the calcineurin B1 subunit $(\mathrm{cnb} 1)$ in the $\mathrm{CD} 11 \mathrm{c}^{+}$population $\left(\mathrm{CD} 11 \mathrm{c}^{\mathrm{cre}}\right)$. Our findings demonstrate that increased susceptibility to A. fumigatus is dependent on calcineurin signaling in myeloid cells. Furthermore, we correlate this increased susceptibility with an observed downregulation of Ptx3 expression in calcineurin-deficient mice. Our data suggest that impairment of antifungal function in myeloid cells through the administration of calcineurin inhibitors, such as CsA and FK506, may be an important factor in the increased susceptibility to IA in immunosuppressed patients.

\section{RESULTS}

Impaired calcineurin signaling in DCs alters expression of genes related to innate immune function

To achieve conditional deletion of calcineurin in DCs, we crossed mice expressing Cre recombinase under control of the $\mathrm{CD} 11 \mathrm{c}$ promoter $\left(\mathrm{CD} 11 \mathrm{c}^{\mathrm{cre}}\right)^{33}$ with mice carrying a floxed allele of the calcineurin regulatory subunit $\mathrm{B}\left(\mathrm{cnb} 1^{\text {loxP }}\right)$ to generate $\mathrm{CD} 11 \mathrm{c}^{\mathrm{cre}} \mathrm{Cnb} 1^{\text {loxP }}$ mice. ${ }^{34}$ Prior to performing a global gene expression analysis to identify calcineurin-regulated functional changes in DCs, we first confirmed $c n b 1$ depletion in $\mathrm{CD} 11 \mathrm{c}^{\mathrm{cre}} \mathrm{cnb} 1^{\text {loxP }} \mathrm{BM}$-derived dendritic-like cells (BMDCs) by quantitative PCR (Figure 1a). We also analyzed BMDCs for expression of IL-2 mRNA (Figure 1b) and IL-2 protein (Figure 1c) upon whole-glucan particles (WGP) (a particulate form of $\beta$-glucan) stimulation. We observed a significant decrease in IL-2 production in calcineurin-impaired cells. IL-2 expression is strictly regulated by the calcineurin-NFAT pathway; therefore, it was used to ensure functional impairment of calcineurin-NFAT signaling in the knockout mice. BMDCs from knockout mice did not display any significant differences in major maturation markers compared with littermate controls following a 6-h stimulation with a panel of pattern recognition receptor ligands as assessed by the percentage positivity and intensity of surface expression of the maturation markers, CD86 (Figures 1d, e) and MHCII (Figures 1f, g), using flow cytometry (see also Supplementary Figure S1 online).

In summary, we show that BMDCs cultured from $\mathrm{CD} 11 \mathrm{c}^{\mathrm{cre}} \mathrm{cnb} 1^{\text {loxP }}$ mice are capable of upregulating major maturation markers upon stimulation with pattern recognition receptors, but exhibit a significant downregulation in the expression of calcineurin-dependent proteins, such as IL-2.

Mice lacking calcineurin in CD11c-expressing cells show
increased susceptibility to systemic $A$. fumigatus infections Based on our previous findings, we hypothesize that a lack of cnb1 in myeloid cells will have an effect on innate immune responses. Therefore, we tested if this is reflected in an increased 

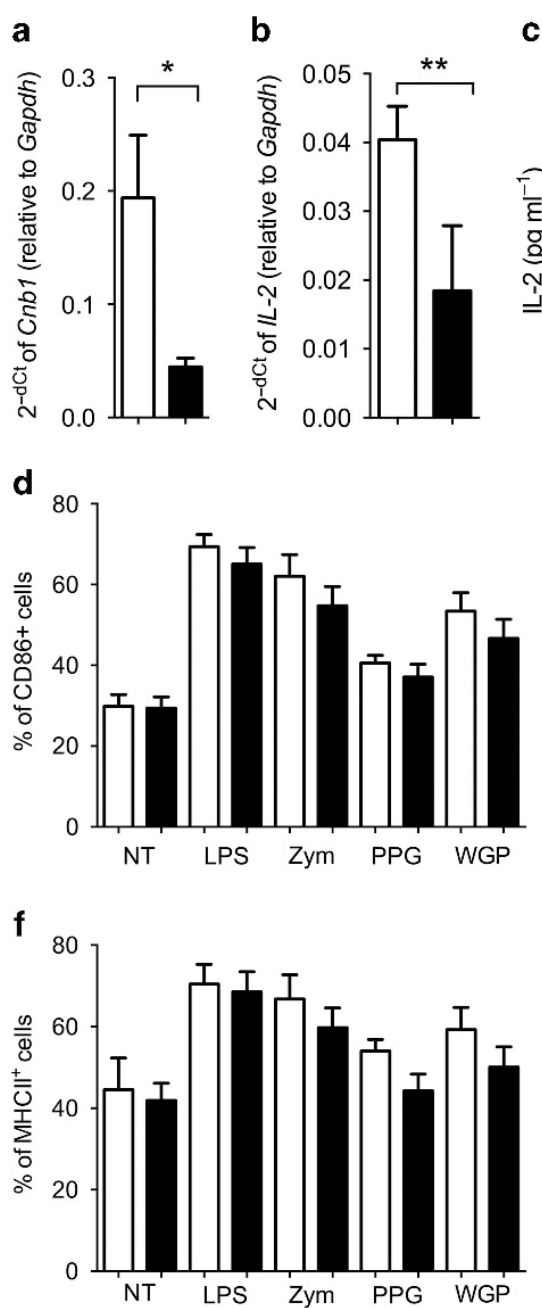
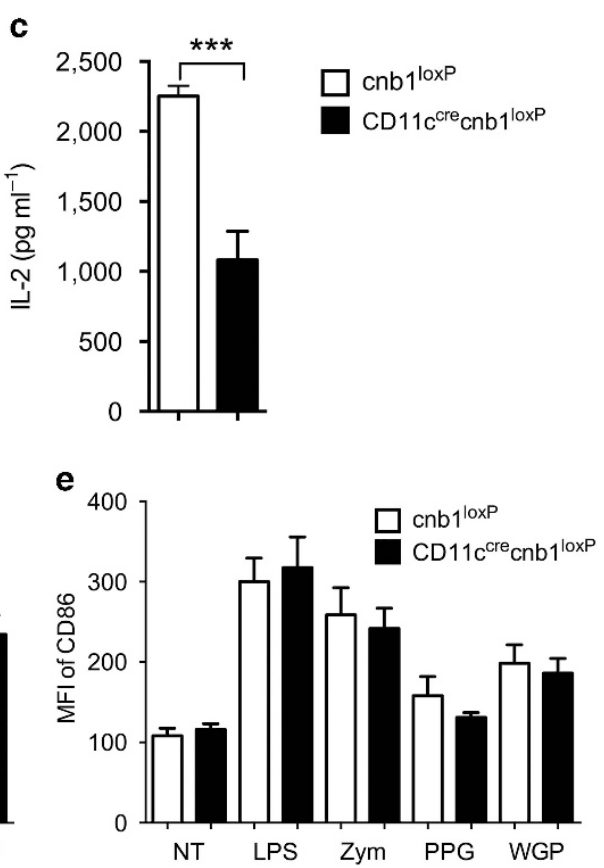

g

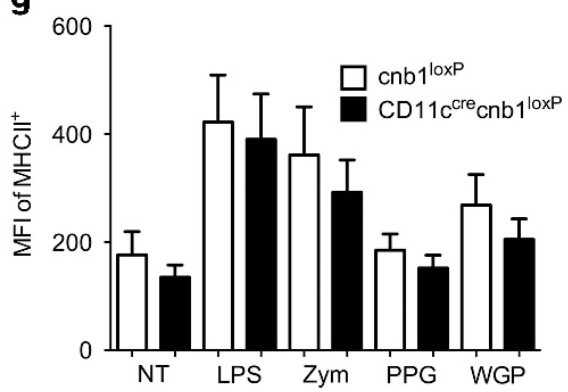

Figure 1 Calcineurin and interleukin-2 (IL-2) expression and maturation capability of bone marrow-derived dendritic-like cells (BMDCs) from $\mathrm{CD} 11 \mathrm{c}^{\text {cre }} \mathrm{Cnb} 1^{\text {loxP }}$ mice. (a) Relative expression of the calcineurin subunit cnb1 mRNA in BMDCs from mice with conditional calcineurin deficiency in CD11c-expressing cells $\left(\mathrm{CD} 11 \mathrm{c}^{\mathrm{cre}} \mathrm{Cnb} 1^{\text {loxP }}\right)$ and their littermate controls $\left(\mathrm{Cnb} 1^{\mathrm{loxP}}\right)$. (b, c) Expression of calcineurin-NFAT-dependent cytokine IL-2

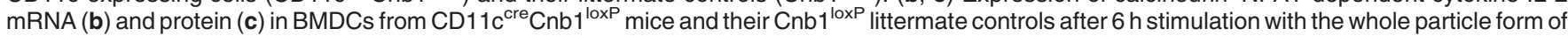
$\beta$-glucan (WGP, whole-glucan particles). (d-g) Maturation of BMDCs after stimulation with a panel of pattern recognition receptor ligands. Percentage (d) as well as mean fluorescence intensity (MFI) (e) of CD86 expression, and percentage (f) and MFI (g) of MHCIl expression in BMDCs from $\mathrm{CD} 11 \mathrm{c}^{\text {cre }} \mathrm{Cnb} 1^{\text {loxP }}$ mice and their $\mathrm{Cnb} 1^{\text {loxP }}$ littermate controls. Pattern recognition receptor ligands are as follows: Untreated (NT), lipopolysaccharide (LPS), Zymozan (Zym), soluble $\beta$-glucan (PPG) particulate form of $\beta$-glucan (WPG). Statistically significant differences between groups are indicated $\left({ }^{\star} P<0.05 ;{ }^{* *} P<0.01 ;{ }^{* *} P<0.001\right)$.

susceptibility to infection with A. fumigatus in vivo. We initially assessed the level of calcineurin depletion in the main myeloid subsets of the CD11 $c^{\text {cre }}$ cnb1 $1^{\text {loxP }}$ mice-DCs, macrophages, and granulocytes. Depletion of $c n b 1$ was confirmed by quantitative PCR in splenic CD11 ${ }^{+}$cells (Figure 2a) and in CD11c + lung myeloid cells (Figure 2b). Calcineurin deficiency was not detected in the control $\mathrm{Gr}^{+}{ }^{+} \mathrm{CD} 11 \mathrm{c}^{-}$granulocyte population (Figure 2b). $\mathrm{CD} 11 c^{\text {cre }} \mathrm{Cnb} 1^{\text {loxP }}$ knockout mice infected intravenously with A. fumigatus showed increased mortality over the 26-day experimental period compared with their littermate controls $\left(\mathrm{Cnb}^{1 \text { loxP }}\right)$ (Figure 2c), which was correlated with a higher fungal load in the infected kidney (Figure 2d). We did not observe any significant changes between infected $\mathrm{CD} 11 \mathrm{c}^{\text {cre }} \mathrm{Cnb}^{\text {loxP }}$ and control mice in total cell number (see Supplementary Figure S2) or in the percentage of myeloid cells in the BM, spleen or lungs (Figures $2 \mathbf{e}-\mathbf{g}$ ).

To understand the involvement of a wider range of myeloid subsets in calcineurin signaling, we further crossed cnb1 $1^{\text {loxP }}$ mice with Cre recombinase under the lysozyme M promoter, LysM $^{\text {cre }}{ }^{35}$ This generates calcineurin deletion in additional myeloid cell subsets, including granulocytes, and hence formed an important additional control. In $\mathrm{LysM}^{\mathrm{cre}} \mathrm{Cnb} 1^{\text {loxP }}$ mice, knockout efficiency was tested in myeloid cells expressing Gr1 isolated from BM (Figure 3a) and in subsets of lung myeloid cells (Figure 3b). Consistent with the previous results, mice infected intravenously with conidia of A. fumigatus showed increased mortality (Figure 3c), which was correlated with a higher fungal load in the infected kidney (Figure 3d), 
a

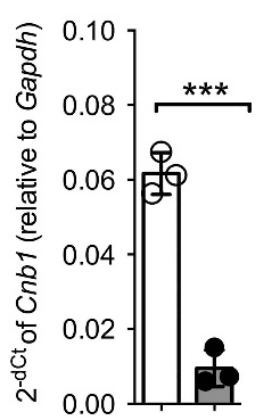

Splenic CD11c ${ }^{+}$cells

b

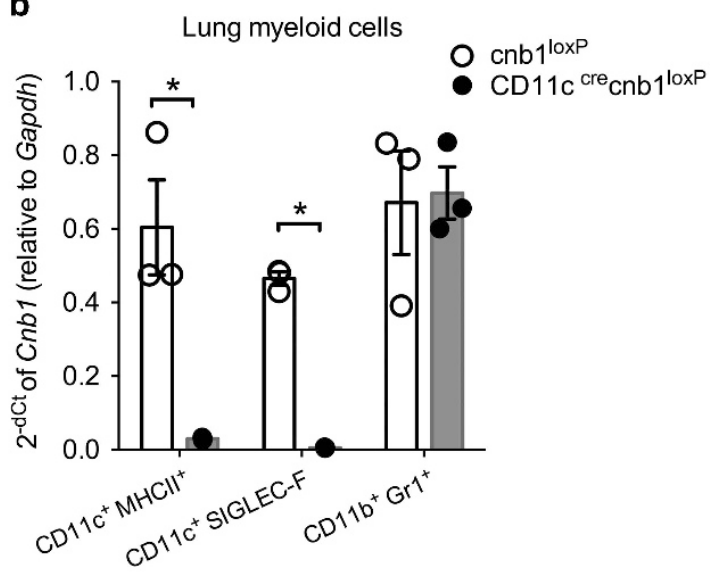

d Kidney CFU (D5)

Survival analysis
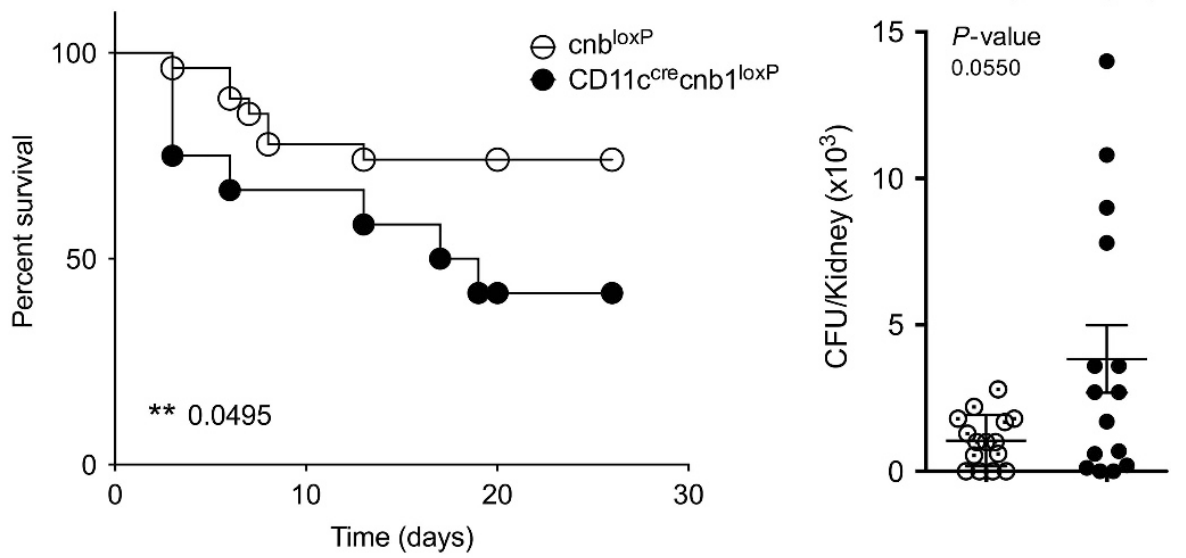

e f
Spleen

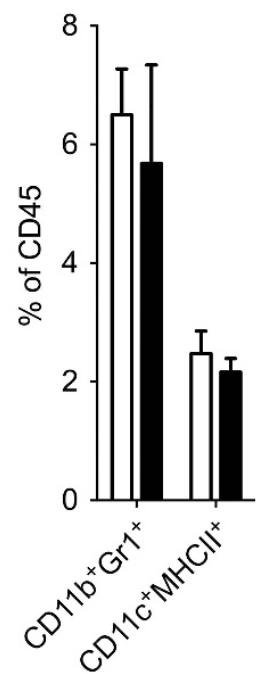

g

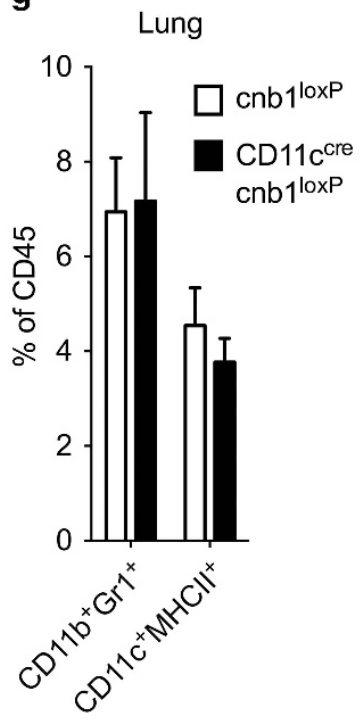

Figure 2 Mice lacking calcineurin in CD11c-expressing cells show increased susceptibility to invasive aspergillosis. (a, b) Efficiency of calcineurin deletion determined as the relative change in cnb1 mRNA expression in: (a) splenic dendritic cells (DCs) (CD11 ${ }^{+}$) from CD11 $\mathrm{c}^{\text {cre }} \mathrm{Cnb} 1^{10 x P}$ mouse and (b) sorted lung myeloid cells from $\mathrm{CD} 11 \mathrm{c}^{\mathrm{cre}} \mathrm{Cnb} 1^{\text {loxP }}$ mouse. Subsets were sorted as following $\mathrm{CD} 11 \mathrm{c}^{+} \mathrm{MHCII}^{+}$as DC-like cells, $\mathrm{CD} 11 \mathrm{~b}{ }^{+} \mathrm{CD} 206^{+}$or SiglecF ${ }^{+}$cells as macrophages and $\mathrm{CD} 11 \mathrm{~b}^{+} \mathrm{Gr} 1^{+}$myeloid cells. $(\mathbf{c}-\mathbf{g}) \mathrm{CD} 11 \mathrm{c}^{\mathrm{cre}} \mathrm{Cnb} 1^{\text {loxP }}$ mice and their cnb1 ${ }^{\text {loxP }}$ littermate controls were inoculated with A. fumigatus (intravenously) to induce systemic aspergillosis. Survival (c) and analysis of fungal load in the lung (CFU: colony-forming units) (d). Cell subset phenotypes in bone marrow (e), spleen (f), and lungs (g) were analyzed. Representative figures of one of three experiments with 3-5 mice per experimental group $(\mathbf{a}, \mathbf{b}, \mathbf{e}, \mathbf{f}, \mathbf{g}) ;(\mathbf{c}, \mathbf{d})$ presenting all data $n=15$. Statistically significant differences between groups are indicated $\left({ }^{\star} P<0.05 ;{ }^{* \star} P<0.01\right.$; $\left.{ }^{* \star \star} P<0.001\right)$. 

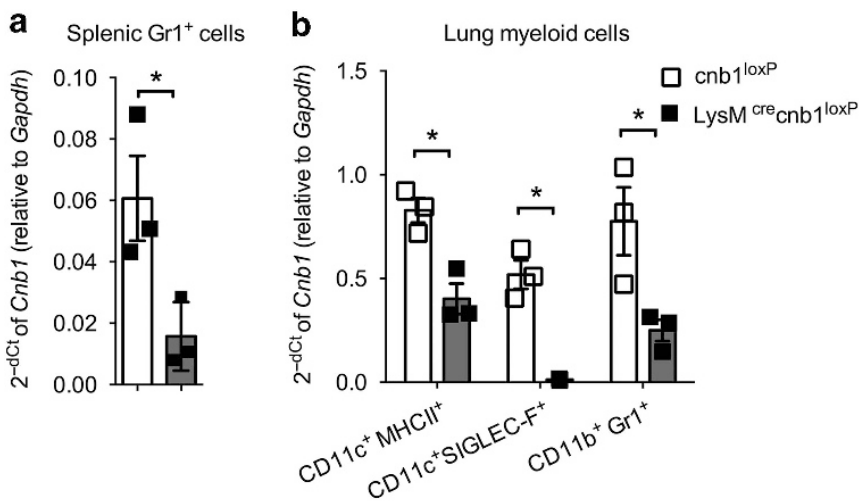

C

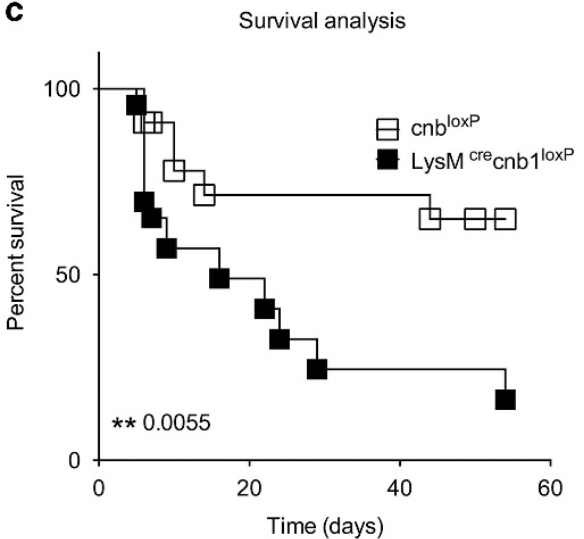

d $\quad$ Kidney CFU (D5)

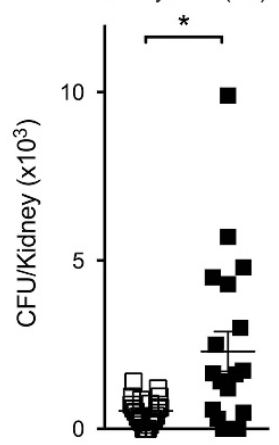

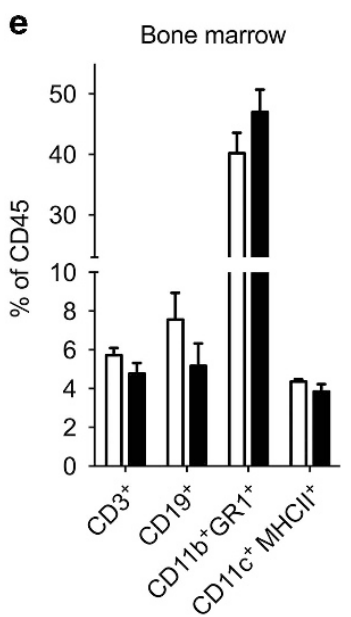

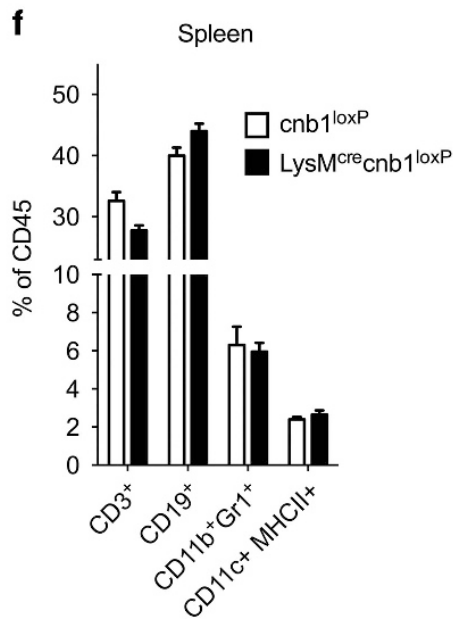

Figure 3 Mice lacking calcineurin in lysozyme M (LysM)-expressing cells show increased susceptibility to invasive aspergillosis. (a, b) Efficiency of calcineurin deletion determined as the relative change in cnb1 mRNA expression in (a) bone marrow (BM)-isolated neutrophils (GR-1 ${ }^{+}$) from LysM ${ }^{\text {ree }} \mathrm{Cnb} 1^{\text {loxP }}$ mouse and (b) sorted lung myeloid cells from LysM ${ }^{\text {cre }} \mathrm{Cnb} 1^{\text {loxP }}$ mice. (c-f) LysM ${ }^{\text {cre }} \mathrm{Cnb} 1^{\text {loxP }}$ mice and their cnb ${ }^{\text {loxP }}$ littermate controls were inoculated with $A$. fumigatus (intravenously) to induce systemic aspergillosis. Survival (c) and analysis of fungal load in the kidney (CFU: colonyforming units). (d) Cell subset phenotypes in bone marrow (e) and spleen (f) were analyzed. Representative figures of one of four experiments with 3-5 mice per experimental group (a, b, e, f); (c, d) presenting all data $n=15-20$. Statistically significant differences between groups are indicated $\left({ }^{\star} P<0.05 ;{ }^{* *} P<0.01 ;{ }^{* *} P<0.001\right)$.

while, as before, no significant differences in the percentage of immune cells in the BM (Figure 3e) and spleen (Figure 3f) were detected.

These experiments demonstrate a key immunoprotective role of calcineurin signaling in CD11c- and LysM-expressing myeloid cells in response to systemic aspergillosis.

\section{Calcineurin signaling in $\mathrm{CD}_{11 c^{+}}$donor cells has an important protective role against invasive pulmonary aspergillosis (IPA) during GvHD}

Uncontrolled A. fumigatus infections are most severe in patients following hematopoietic stem cell or BM transplant and treatment with immunosuppressive drugs. These drugs, 


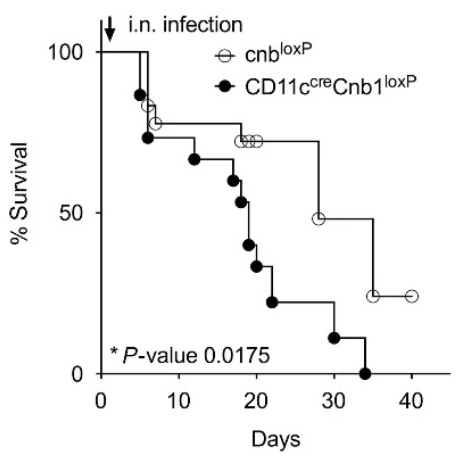

b

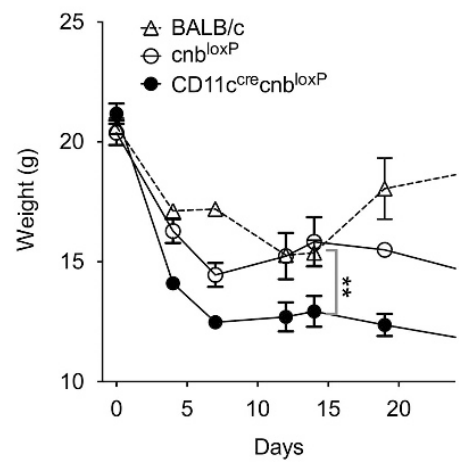

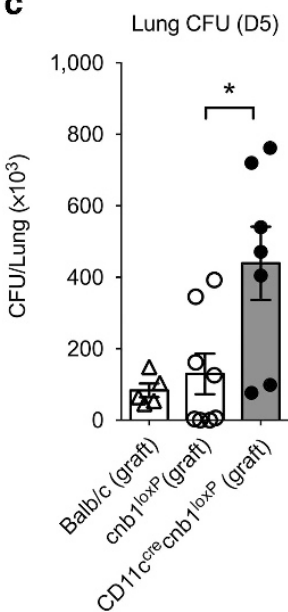

Figure 4 Calcineurin in CD11c-expressing cells enhance protection against invasive pulmonary aspergillosis in mice with allograft bone marrow transplant. (a-c) Irradiated BALB/c mice were engrafted with bone marrow from CD $11 c^{\text {cre }} \mathrm{cnb} 1^{\text {loxP }}$ and cnb $1^{\text {loxP }}$ donors. Mice were inoculated (intranasally) with $A$. fumigatus one day post-transplant. Survival (a) and body weight (b) were analyzed. Some mice were killed on day 5 for analysis of fungal load (CFU: colony-forming units) (c). Representative figures of one of three experiments with 3-5 mice per experimental group are shown. Statistically significant differences between groups are indicated $\left({ }^{*} P<0.05 ;{ }^{* *} P<0.01\right)$.

which are typically calcineurin NFAT inhibitors (e.g., CsA and FK506), are used to control immune reactions of the transplanted cells, that is, GvHD or transplant rejection, respectively. Because they are administered systemically, specific direct effects on myeloid cells are difficult to analyze.

Here, we were interested in how an intrinsic lack of calcineurin signaling in donor cells expressing CD11c influences protection against IPA in irradiated and $\mathrm{BM}$-engrafted mice. Using a mouse model of GvHD, host $\mathrm{BALB} / \mathrm{c}$ mice were irradiated and engrafted with allogeneic $\mathrm{BM}$ from $\mathrm{CD} 11 \mathrm{c}^{\mathrm{cre}} \mathrm{Cn}$ $\mathrm{b} 1^{\text {loxP }}$ or $\mathrm{Cnb1} 1^{\text {loxP }}$ control donors. Three days post $\mathrm{BM}$ transplantation, all mice were intranasally infected with $A$. fumigatus. Significantly increased mortality was observed in mice engrafted with $\mathrm{CD} 11 \mathrm{c}^{\mathrm{cre}} \mathrm{Cnb} 1^{\text {loxP }}$ compared with control Cnb1 ${ }^{\text {loxP }}$ BM (Figure 4a), and the higher susceptibility to $A$. fumigatus infection was also apparent in the increased weight loss following donor cell engraftment (Figure $\mathbf{4 b}$ ). Mortality and weight loss correlated with a higher fungal load in the lungs of mice engrafted with $\mathrm{CD} 11 c^{\text {cre }} \mathrm{Cnb}^{\text {loxP }} \mathrm{BM}$ compared with Cnb1 ${ }^{\text {loxP }}$ BM (Figure 4c).

In summary, we show that mice engrafted with BM from donors with impaired calcineurin signaling in CD11c-expressing cells are more susceptible to A. fumigatus infection. This result may explain the difficulties associated with controlling disseminated A. fumigatus infections during GvHD in patients undergoing treatment with the calcineurin inhibitors, CsA and FK506.

\section{Calcineurin signaling regulates expression of a key molecule in fungal defense-pentraxin-3}

We performed a global gene expression analysis to identify calcineurin-regulated functional changes in $\mathrm{CD} 11 \mathrm{c}^{+}$myeloid cells. BMDCs from $\mathrm{CD} 11 \mathrm{c}^{\mathrm{cre}} \mathrm{cnb} 1^{\text {loxP }}$ mice and their littermate controls, in which calcineurin signaling was functional $\left(\mathrm{cnb1} 1^{\operatorname{loxP}}\right)$, were stimulated with A. fumigatus for $6 \mathrm{~h}$. The
$\mathrm{CD}_{11 \mathrm{c}^{+}}$cell preparations were first depleted of $\mathrm{Gr} 1^{+}$cells and a phenotypic analysis is shown in Supplementary Figure S3 for each preparation. Three different morphotypes of A. fumigatus, conidia, swollen conidia, and hyphae, were used, which represent the different biological forms of A. fumigatus. As the fungus progresses through its life cycle during infection, it exposes different surface amounts of $\beta$-glucan, which trigger different signaling pathways.

Although CD11 $c^{\text {cre }} \mathrm{cnb} 1^{\text {loxP }}$ BMDCs displayed no significant changes in major maturation markers (Figures 1d-g), global expression analysis by two-way analysis of variance following a 6-h stimulation with A. fumigatus morphotypes revealed a number of functional changes in gene expression. A total of 188 probes were significantly altered in expression between wild type and knockout mice after multiple testing correction (see Supplementary Figure S4 and Supplementary Tables S1 and S2). A MetaCore enrichment analysis of these differentially expressed genes was also performed. Table 1 shows a summary of the most significantly enriched 'Process networks' (A), 'Pathway Maps' (B), and 'GO processes' (C). A full list of the pathways is listed in Supplementary Table S1. The enrichment analysis suggests strong changes in innate immune responses, including several inflammatory pathways. The analysis also identified several differentially expressed genes responsible for innate responses (see Supplementary Table S2). These included pentraxin-3, a molecule of particular importance in antifungal activity, which was expressed at significantly lower levels in BMDCs from mice lacking calcineurin in CD11c-expressing cells $\left(\mathrm{CD} 11 \mathrm{c}^{\mathrm{cre}} \mathrm{cnb} 1^{\text {loxP }}\right)$. Heat-map analysis (Figure 5a) shows the differentially expressed genes associated with Gene Ontology terms relevant to infection: response to yeast, inflammatory response, opsonization and negative regulation by host. 
Table 1 Global gene expression analysis of BMDCs from CD11c ${ }^{\text {cre }}$ cnb1 ${ }^{\text {loxP }}$ mice and their cnb1 ${ }^{\text {loxP }}$ littermate controls, stimulated with three different morphotypes of $A$. fumigatus

P-value

\begin{tabular}{|c|c|c|}
\hline (A) & Enrichment by process networks & \\
\hline 1 & Reproduction: feeding and neurohormone signaling & 0.01577 \\
\hline 2 & Proliferation: positive regulation cell proliferation & 0.0193 \\
\hline 4 & Inflammation: interferon signaling & 0.02137 \\
\hline 5 & Development: blood vessel morphogenesis & 0.02223 \\
\hline 8 & Inflammation: Jak-STAT pathway & 0.03328 \\
\hline 9 & Immune response: innate immune response to RNA viral infection & 0.04855 \\
\hline (B) & Enrichment by pathway maps & \\
\hline 1 & Immune response: IL-3 activation and signaling pathway & 0.00149 \\
\hline 5 & Role of endothelin-1 in inflammation and vasoconstriction in sickle cell disease & 0.00271 \\
\hline 6 & Transport: RAB1A regulation pathway & 0.00339 \\
\hline 7 & Development: oligodendrocyte differentiation from adult stem cells & 0.00625 \\
\hline 8 & Immune response: antiviral actions of interferons & 0.00660 \\
\hline 9 & Mechanism of action of CCR4 antagonists in asthma and atopic dermatitis & 0.00739 \\
\hline 10 & Transcription: assembly of RNA Polymerase II preinitiation complex on TATA-less promoters & 0.00763 \\
\hline 11 & Development: c-Kit ligand signaling pathway during hemopoiesis & 0.01025 \\
\hline 12 & Expression targets of tissue factor signaling in cancer & 0.01131 \\
\hline 13 & Immune response: MIF-mediated glucocorticoid regulation & 0.01131 \\
\hline 4 & Response to organic substance & 5.20E-09 \\
\hline 5 & Negative regulation of cellular process & 7.46E-09 \\
\hline 6 & Immune response & 8.78E-09 \\
\hline 7 & Negative regulation of biological process & $1.85 \mathrm{E}-08$ \\
\hline 8 & Regulation of response to stimulus & 2.80E-08 \\
\hline 9 & Negative regulation of cell activation & 5.31E-08 \\
\hline 10 & Negative regulation of exocytosis & 7.33E-08 \\
\hline
\end{tabular}

Bone marrow-derived dendritic-like cells were prepared from mice lacking cnb1 in cells expressing CD11c and their littermate controls. Cells were stimulated for $6 \mathrm{~h}$ with conidia, swollen conidia and hyphae of A. fumigatus. Enrichment analysis of differentially expressed genes, selected using ANOVA, was performed via hypogeometric enrichment analysis of the differentially expressed genes using GeneGo (www.genego.com). Table 1 and Supplementary Table S1 online show Process networks (A), Pathway Maps (B), and GO processes (C).

To understand the molecular mechanism underlying an increased susceptibility to A. fumigatus infection in calcineurin-deficient myeloid cells, we evaluated the differentially expressed genes identified by the global expression analysis described above (see Supplementary Table 2) for evidence of known antifungal activities. This analysis identified pentraxin-3, which is produced in many subsets of myeloid cells, although no link to calcineurin-NFAT signaling has been reported previously. To validate the observed downregulation of Ptx3 in calcineurin-depleted $\mathrm{CD}_{11 \mathrm{c}^{+}}$cells in our global gene 


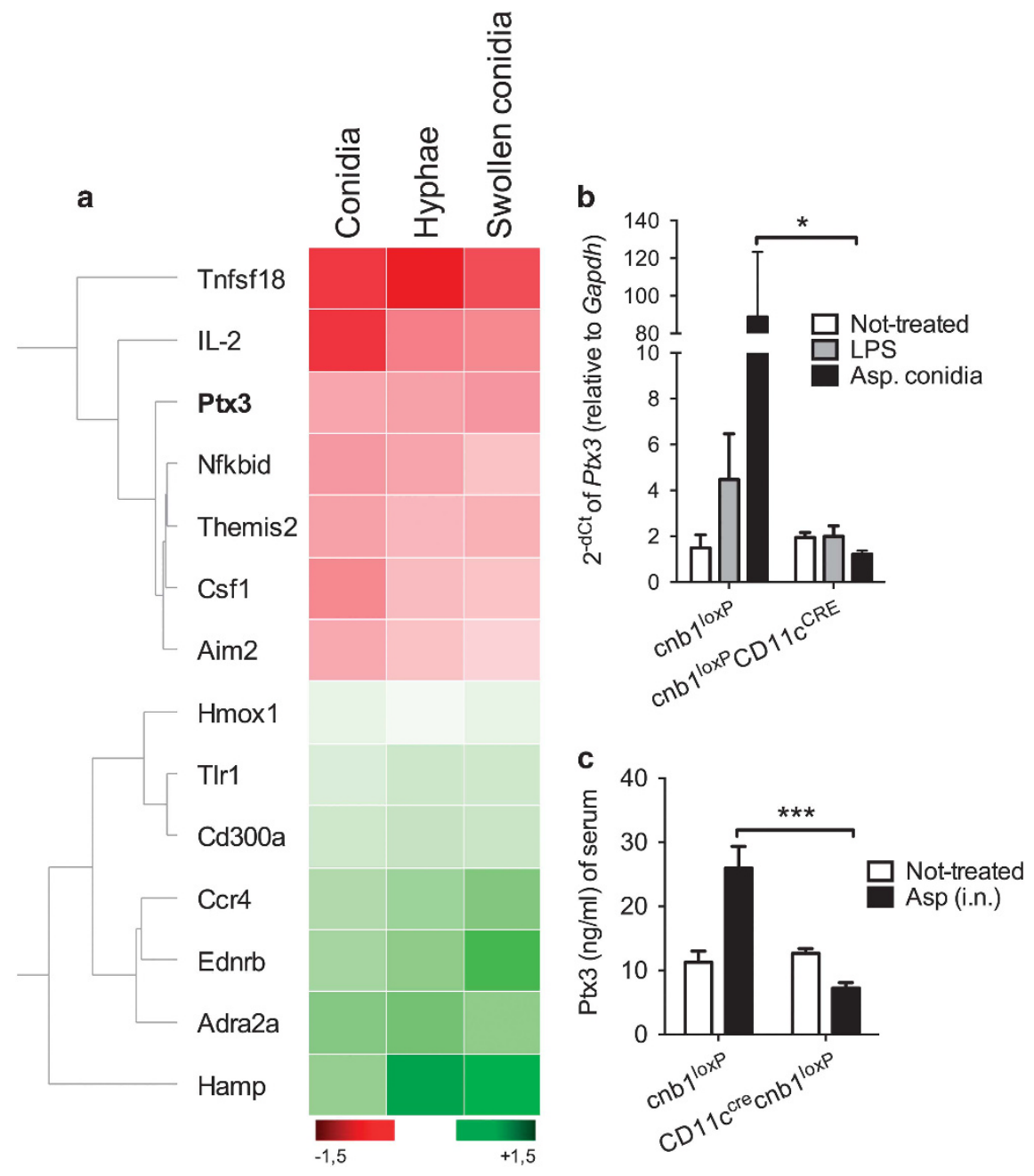

Figure 5 Global gene expression analysis of calcineurin-deficient BMDCs. (a) Heat map of gene expression of BMDCs from CD11 $\mathrm{c}^{\mathrm{cre}} \mathrm{cnb} 1^{\text {loxP }}$ mice and their $\mathrm{cnb} 1^{\text {loxP }}$ littermate controls, stimulated with three different morphotypes of $A$. fumigatus. Bone marrow-derived dendritic-like cells were prepared from mice lacking $\mathrm{cnb} 1$ in cells expressing CD11c and their littermate controls $(n=3)$. Cells were stimulated for $6 \mathrm{~h}$ with conidia, swollen conidia, and hyphae of $A$. fumigatus. Heat map depicts the log2 fold changes of the differentially expressed genes associated with Gene Ontology terms: response to yeast, inflammatory response, opsonization and negative regulation by host. The log2 fold changes are colored red to green with no change being white (fold change range of -0.99 to 1.32). (b) Relative expression of Ptx3 mRNA in bone marrow-derived dendritic-like cells (BMDCs) from mice with conditional calcineurin deficiency in CD11c-expressing cells $\left(\mathrm{CD} 11 \mathrm{c}^{\mathrm{cre}} \mathrm{Cnb} 1^{\text {loxP }}\right)$ and their littermate controls $\left(\mathrm{Cnb} 1^{\text {loxP }}\right)$, Gr1 ${ }^{+}$cells were depleted using magnetic beads. $N=3$, statistically significant differences between groups are indicated $\left({ }^{\star} P<0.05\right)$. (c) $C D 11 c^{\text {cre }} \mathrm{cnb}^{\text {loxP }}$ mice and their $\mathrm{cnb} 1^{\text {loxP }}$ littermate controls ( $\mathrm{Cnb} 1^{\mathrm{loxP}}$ ) were inoculated with $A$. fumigatus (intranasally) to induce invasive pulmonary aspergillosis. Levels of serum Ptx 3 were analyzed $48 \mathrm{~h}$ after inoculation by ELISA. $N=5$, statistically significant differences between groups are indicated $\left({ }^{\star \star \star} P<0.001\right)$.

transcription analysis, we evaluated Ptx3 expression in calcineurin-depleted BMDCs. We observed a significant decrease in Ptx3 mRNA expression in BMDCs stimulated with conidia of $A$. fumigatus (Figure 5b). Gr1 ${ }^{+}$cells were first eliminated by magnetic bead depletion, resulting in a high purity of $\mathrm{CD}_{11 \mathrm{c}^{+}}$cells in these preparations (see Supplementary Figure S3). We also investigated changes in Ptx3 expression in vivo in $\mathrm{CD} 11 \mathrm{c}^{\text {cre }} \mathrm{Cnb} 1^{\text {loxP }}$ mice following intravenous injection of $A$. fumigatus conidia. Sera from untreated and intravenously infected control $\left(\mathrm{Cnb}^{\mathrm{loxP}}\right)$ and knockout $\left(\mathrm{CD} 11 \mathrm{c}^{\text {cre }} \mathrm{Cnb} 1^{\text {loxP }}\right)$ mice were sampled $24 \mathrm{~h}$ after infection. A significant drop in Ptx3 levels in infected mice lacking calcineurin under the $\mathrm{CD} 11 \mathrm{c}$ promoter could be observed (Figure 5c).
In vitro depletion of $C n b 1$ in myeloid cells was not absolute in $\mathrm{CD} 11 \mathrm{c}^{\text {cre }} \mathrm{Cnb} 1^{\text {loxP }}$ mice (Figure 1a); therefore, we induced calcineurin knockdown in $\mathrm{Mxl}^{\text {cre }}{ }^{\mathrm{Cnb}} 1^{\text {loxP }}$ mice, in which injection with Poly I:C activates Cre recombinase under the control of a type I interferon inducible promoter $\left(\mathrm{Mx}^{\mathrm{cre}}\right)$. This model provides efficient deletion of calcineurin in vivo in all hematopoietic cells, including BMDCs, which is maintained after stimulation with WGP or A. fumigatus conidia (see Supplementary Figure S5a). We observed a significant decrease in Ptx3 mRNA expression in BMDCs from $\mathrm{Mx}^{\text {cre }}{ }^{\text {Cnb }} 1^{\text {loxP }}$ mice in steady-state conditions as well as upon stimulation with WGP or A. fumigatus conidia (see Supplementary Figure S5b). Accordingly, decreased levels of Ptx3 protein were also observed in supernatants of BMDCs 
from $\mathrm{Mx} 1^{\text {cre }} \mathrm{Cnb}_{1}{ }^{\text {loxP }}$ mice compared with littermate controls following stimulation with bacterial lipopolysaccharide: the viral mimetic, Poly I:C; WGP; and A. fumigatus conidia (see Supplementary Figure S5c).

Taken together, we show that expression of the potent antifungal protein, Ptx3, is significantly downregulated in BMDCs with impaired calcineurin signaling when stimulated in vitro with a range of pathogen-associated molecular patterns or with A. fumigatus in vitro, and that regulation of Ptx3 through calcineurin signaling is associated with increased susceptibility to A. fumigatus.

\section{DISCUSSION}

This study reveals the critical importance of calcineurin signaling in myeloid cells in the control of fungal infections. We show that specific deletion of calcineurin in DCs causes a significant increase in susceptibility to IA. Furthermore, calcineurin signaling in CD11c-expressing cells from donor $\mathrm{BM}$ was a critical component promoting IPA survival in irradiated recipients. Lack of calcineurin in CD11c-expressing subsets, including spleen and lung myeloid cells, considerably increased susceptibility to A. fumigatus. Our results are of particular significance in the context of the clinical observation that pulmonary aspergillosis is a leading cause of increased mortality in transplant patients. ${ }^{36}$ Treatment with cyclosporine A or tacrolimus may increase patients' susceptibility to A. fumigatus, protection from which requires both innate and adaptive immune responses. ${ }^{37}$ The direct role of calcineurin in myeloid subsets has been long overlooked due to the difficulties in dissecting the roles of specific cell subsets following systemic drug administration. Here, we show that calcineurin signaling in myeloid cells has a key function in innate immune protection.

Herbst et al. ${ }^{14}$ first demonstrated a link between TLR9NFAT signaling and phagocytosis, ${ }^{14}$ supported by our own data showing that calcineurin-NFAT is a major sensor of $\beta$-glucan, a particulate form of pathogen-associated molecular pattern. ${ }^{18}$ Global gene expression analysis of BMDCs lacking calcineurin showed important changes in the expression of genes responsible for innate defense as well as processes of innate immunity involved in the response to fungi. These results encouraged further analysis of the importance of calcineurin signaling in myeloid cells in antifungal protection in vivo. We show that mice deficient in calcineurin expression under the LysM or CD11c promoters were significantly more susceptible to systemic aspergillosis. We therefore speculated that inhibition of calcineurin is also important in the context of increased susceptibility of immunosuppressed transplant patients to opportunistic infections such as invasive fungal pathogens. ${ }^{1,2}$

We infected allogeneic host mice with A. fumigatus 1 day after transplantation of $\mathrm{BM}$ from donors with impaired calcineurin signaling in CD11c-expressing cells. Mice were infected intranasally to mimic IPA. BALB/c mice engrafted with BM from allogeneic mice lacking calcineurin in CD11cexpressing cells were less able to control Aspergillus infection and showed significantly higher mortality than calcineurinsufficient controls.
To determine the mechanism of this increased susceptibility, we interrogated a global gene expression analysis of calcineurin-impaired DCs for differentially expressed genes known to affect antifungal immunity. This identified Ptx3, which has been previously described as a key molecule in immune protection to A. fumigatus $^{24}$ and other infections. ${ }^{38}$ Ptx3 is known to exert a broad range of immune activities from opsonic capacity ${ }^{24}$ to complement activation and phagocytosis, ${ }^{30}$ and stimulation of the TLR4-MD-2 pathway. ${ }^{39}$ During fungal infections, Ptx3 expression is activated in DCs through TLR activation and NF- $\mathrm{kB},{ }^{39,40}$ and it also forms a component of the neutrophil extracellular traps protein pool. ${ }^{41}$ Here, we report that Ptx3 expression can be co-regulated through the calcineurin-NFAT pathway, suggesting that systemically administered immunosuppressive drugs targeting the calcineurin-NFAT binding might affect Ptx3 production. Follow-up studies investigating this directly in transplant patients would be very useful.

Our findings demonstrate that a lack of calcineurin signaling in various myeloid cells is a critical factor for A. fumigatus infection susceptibility and survival, indicating that an element of the innate response to Aspergillus is calcineurin dependent. This is particularly important in the early stages following allogeneic BM transplantation, where we observed that mice engrafted with calcineurin-deficient CD11c-expressing cells had a significantly higher mortality in the presence of systemic A. fumigatus infection. Our data support previous studies demonstrating a critical role for NFAT in innate antifungal defense, ${ }^{13,14}$ and suggest a possible mechanism of action. Although the role of Ptx3 in antifungal defense and its therapeutic potential for the treatment of IA $^{42,43}$ has been well described, this study is the first, to our knowledge, to describe the co-regulation of Ptx3 through the NFAT pathway. Receptors leading to NFAT activation in DCs, such as TLR4 (refs. 44,45) or Dectin-1 deficiency, ${ }^{46-48}$ have been shown to be essential for aspergillosis susceptibility after BM transplant, which strongly support our findings.

Although A. fumigatus recognition by DCs results in stimulation of a broad range of pattern recognition receptors and triggering of a number of defense mechanisms, including cytokine induction of adaptive immunocytes, we focused on the direct innate antifungal response. We show that calcineurin deficiencies in CD11c- and LysM-positive myeloid cells lead to impaired Ptx3 expression and reduced survival in A. fumigatus infection. Calcineurin signaling in $\mathrm{CD}_{11 \mathrm{c}^{+}}$-expressing spleen and lung myeloid cells therefore provide essential protection from A. fumigatus infections, which may be an important factor in the increased susceptibility described in calcineurin inhibitor-treated transplant patients.

\section{METHODS}

Animals. Mice were purchased from The Jackson Laboratory (Bar Harbor, ME) and maintained under specific pathogen-free conditions. $\mathrm{Mx}^{\text {cre }}{ }^{49} \mathrm{CD} 11 \mathrm{c}^{\text {cre }}$, 3 and $\mathrm{LysM}^{\text {cre }}$ (ref. 35) mice expressing cre recombinase under tissue-specific promoters were crossed with

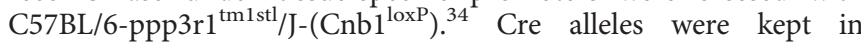


heterozygosis and littermates were used as controls. All knockout and wild-type mice $\mathrm{C} 57 \mathrm{BL} / 6$ and $\mathrm{BALB} / \mathrm{c}$ were used at $8-12$ weeks old. In the GvHD model, $\mathrm{BALB} / \mathrm{c}$ recipients were lethally irradiated with $12 \mathrm{~Gy}$ in two doses separated by $4 \mathrm{~h}$, and reconstituted with 5 million $\mathrm{BM}$ cells from conditional knockout $\mathrm{CD} 11 \mathrm{c}^{\mathrm{cre}} \mathrm{Cnb} 1^{\text {loxP }}$ mice or littermate controls $\left(\mathrm{Cnb} 1^{\mathrm{loxP}}\right)$. To induce $\mathrm{Cnb} 1$ deletion in $\mathrm{Mxl}^{\text {cre }} \mathrm{Cnb}^{\text {loxP }}$ mice, animals were treated with $250 \mu \mathrm{g}$ poly I:C (Invivogen, San Diego, CA), administered by intraperitoneal injection every other day over the course of 10 days. All mice were bred in the Biological Resource Centre (A*STAR, BMRC, Singapore) and handled according to the institute guidelines under the approval of the Institutional Animal Care and Use Committee (IACUC \#110626).

A. fumigatus infection. For induction of IPA, mice were anesthetized and infected intranasally with three doses of $10^{10} \mathrm{~A}$. fumigatus (MYA-4609; ATCC, Manassas, VA) conidia $(20 \mu \mathrm{l})$ over three consecutive days. For induction of IA, intravenous infections were performed by injecting $2.5 \times 10^{6}$ conidia of A. fumigatus $(200 \mu \mathrm{l})$, which were harvested in phosphate-buffered saline/0.05\% Tween 20 after 5 days of cultivation on potato dextrose agar (Sigma Aldrich, St. Louis, MO). Fungal load in organs was analyzed using tissue homogenates cultured in serial dilutions on Sabouraud agar plates, in triplicate, to allow calculation of the mean number of colony-forming units per organ as described. ${ }^{50}$ Preparation of swollen conidia and hyphae was described elsewhere. ${ }^{50}$

Flow cytometry. Single-cell suspensions obtained from spleens and $\mathrm{BM}$ were labeled with anti-mouse antibodies: CD11b (FITC), CD11c (APC), CD45 (PeCy7), CD86 (PE), CD206 (BV605), MHC-II (AF700), Gr-1 (eF450), and SiglecF (PE). For some experiments, myeloid subsets were sorted using an Aria II cell sorter (BD, Franklin Lakes, NJ). Lung suspensions were prepared using collagenase ${ }^{22}$ followed by CD $45^{+}$ magnetic bead enrichment (Miltenyi Biotec, Cologne, Germany). Samples were acquired using an LSRII or MacsQuant and analyzed using FloJo (TreeStar, Ashland, OR).

Cell culture. BM cells were cultured in Iscove's modified Dulbecco's medium (containing $10 \mathrm{ng} \mathrm{ml}^{-1}$ granulocyte-macrophage colonystimulating factor, $10 \%$ heat-inactivated fetal calf serum (Life Technologies, Invitrogene, Carlsbad, CA), $100 \mathrm{mg} \mathrm{ml}^{-1}$ streptomycin, and $100 \mathrm{U} \mathrm{ml}^{-1}$ penicillin) at $5 \times 10^{5}$ cells per ml. On day 6 , cells were harvested and re-stimulated with a panel of TLR ligands: $1 \mu \mathrm{g} \mathrm{ml}{ }^{-1}$ lipopolysaccharide (Alexis), $1 \mu \mathrm{g} \mathrm{ml}^{-1}$ Zymozan (InVivogen), $1 \mu \mathrm{g} \mathrm{ml}^{-1}$ Poly I:C (InVivogen) $1 \mu \mathrm{g} \mathrm{ml}^{-1}$ of soluble (PPG) and whole particles (WGP) of (1-3,1-6)- $\beta$-D-glucan (Wellmune), and A. fumigatus morphotypes (conidia, swollen conidia, and hyphae). Depletion of contaminating Gr-1 positive cells was performed prior to DC stimulation using an AutoMACS (Miltenyi Biotec).

Enzyme-linked immunosorbent assay. Ptx3 ELISA was performed using the DuoSet kit (R\&D Systems, Minneapolis, MN) following the manufacturer's recommendations.

Quantitative real-time PCR. Total cellular RNA was extracted by Trizol (Invitrogen, Carlsbad, CA) phase separation followed by purification using the RNeasy Mini/Micro kit (Qiagen) or the Arcturus PicoPure RNA Isolation Kit. Reverse transcription was carried out using a highcapacity cDNA Reverse Transcription Kit with RNase Inhibitor (Applied Biosystems, Carlsbad, CA) or with SuperScript III First Strand Synthesis System for RT-PCR (Invitrogen). Real-time PCR was carried out using GoTaq qPCR Master Mix (Promega, Madison, WI) with primers: cnb1 (Fw: TCG ACA CAG ACG GCA ACG GA, Rv: GGC CAC CTA CGA CAG CAC AGA), IL-2 (Fw: CCC AGG ATG CTC ACC TTC, Rv CAA CAG TTA CTC TGA TAT TGC TGA TG), and Ptx3 (Fw: CTG CCC GCA GGT TGT GAA A, Rv: ACC AAC ACT AGG GAC TGG GA), and normalized to GAPDH (Fw: TCG TCC CGT AGA CAA AAT GG, Rv: TTG AGG TCA ATG AAG GGG TC). Normalization was performed using the ddCt method.
Microarray hybridization and analysis. Total RNA was extracted in triplicate samples from wild type and knockout mice treated with conidia, swollen conidia, and hyphae using a double extraction protocol. Fragmented ssDNAs were hybridized to the standard arrays for $17 \mathrm{~h}$ at $45^{\circ} \mathrm{C}$. The arrays were washed and stained using a fluidics station, and then scanned using the GeneChip Scanner 3000 (Affymetrix, Santa Clara, CA). Gene expression data generated on Affymetrix GeneChip Mouse Gene ST arrays were processed using Bioconductor version 2.11 (www.bioconductor.org, Seattle, WA), running on $\mathrm{R}$ version 2.15.2. Normalization of the arrays was performed using the robust multi-array average, and the resulting log2transformed values were used for all subsequent analysis. The gene expression data were then filtered for probes where the associated gene had a valid NCBI Entrez Gene ID in order to restrict data to wellannotated genes. The data were also filtered to ensure an interquartile range greater than 0.5 . A series of two-way analysis of variances was used to identify probes for which the factors, treatment, knockout status or the interaction between treatment and knockout status, had a significant effect. Multiple test corrections were applied using the method of Benjamini and Hochberg. Processing of the data was carried out within a workflow created in Biovia Pipeline Pilot and the data were visualized in TIBCO Spotfire. All microarray data files are available for free download at the Gene Expression Omnibus (GEO accession number: GSE71936 'http://www.ncbi.nlm.nih.gov/geo'). MetaCore from Thomson Reuters (New York, NY) was used for the enrichment analysis.

Statistical analysis. Unless specified differently in the legend, all values are shown as means \pm s.e.m. Unpaired Student's $t$-test was used to identify significant differences between groups. For all tests, the 0.05 confidence level was considered statistically significant. In figures ${ }^{*}$ denotes $P<0.05$, ${ }^{*}$ denotes $P<0.01$, and ${ }^{* *}$ denotes $P<0.001$. $P$-values in survival experiments were determined using the log-rank test (Mantel-Cox); exact $P$-values are stated in the graphs.

SUPPLEMENTARY MATERIAL is linked to the online version of the paper at $\mathrm{http}: / /$ www.nature.com/mi

\section{ACKNOWLEDGMENTS}

We thank C. Phua and S. Nabti for animal handling, I. Low and N. Shadan from SlgN flow cytometry facility for cell sorting, H.S. Tay for technical assistance, and Kerry McLaughlin from Insight Editing London for review of the manuscript. This work was supported by the BMRC, A*STAR, Singapore and the project no. LQ1605 from the National Program of Sustainability II (MEYS CR) and by the project FNUSA-ICRC no. CZ.1.05/ 1.1.00/02.0123 (OP VaVpl).

\section{DISCLOSURE}

The authors declared no conflict of interest.

(c) 2017 Society for Mucosal Immunology

\section{REFERENCES}

1. Fishman, J.A. Infections in immunocompromised hosts and organ transplant recipients: essentials. Liver Transpl. 17 (Suppl 3), S34-S37 (2011).

2. Pappas, P.G. et al. Invasive fungal infections among organ transplant recipients: results of the Transplant-Associated Infection Surveillance Network (TRANSNET). Clin. Infect. Dis. 50, 1101-1111 (2010).

3. Crassard, N. et al. Invasive aspergillosis and allogeneic hematopoietic stem cell transplantation in children: a 15-year experience. Transpl. Infect. Dis. 10, 177-183 (2008).

4. Kontoyiannis, D.P. et al. Prospective surveillance for invasive fungal infections in hematopoietic stem cell transplant recipients, 2001-2006: overview of the Transplant-Associated Infection Surveillance Network (TRANSNET) Database. Clin. Infect. Dis. 50, 1091-1100 (2010).

5. Upton, A., Kirby, K.A., Carpenter, P., Boeckh, M. \& Marr, K.A. Invasive aspergillosis following hematopoietic cell transplantation: outcomes and 
prognostic factors associated with mortality. Clin. Infect. Dis. 44, 531-540 (2007).

6. Pagano, L. et al. Invasive aspergillosis in patients with acute myeloid leukemia: a SEIFEM-2008 registry study. Haematologica 95, 644-650 (2010).

7. Marotta, G. et al. Complete resolution of hepatic aspergillosis after nonmyeloablative hematopoietic stem cell transplantation in a patient with acute myeloid leukemia. Hematology 10, 383-386 (2005).

8. Herbst, S. et al. A new and clinically relevant murine model of solid-organ transplant aspergillosis. Dis. Model Mech. 6, 643-651 (2013).

9. Steinmann, J. et al. Emergence of azole-resistant invasive aspergillosis in HSCT recipients in Germany. J. Antimicrob. Chemother. 70, 1522-1526 (2015).

10. Fric, J. et al. NFAT control of innate immunity. Blood 120, 1380-1389 (2012).

11. Vandewalle, A., Tourneur, E., Bens, M., Chassin, C. \& Werts, C. Calcineurin/NFAT signaling and innate host defence: a role for NOD1mediated phagocytic functions. Cell Commun. Signal. 12, 8 (2014).

12. Romani, L. Immunity to fungal infections. Nat. Rev. Immunol. 11, 275-288 (2011).

13. Greenblatt, M.B., Aliprantis, A., Hu, B. \& Glimcher, L.H. Calcineurin regulates innate antifungal immunity in neutrophils. J. Exp. Med. 207, 923-931 (2010).

14. Herbst, S. et al. Phagocytosis-dependent activation of a TLR9-BTKcalcineurin-NFAT pathway co-ordinates innate immunity to Aspergillus fumigatus. EMBO Mol. Med. 7, 240-258 (2015).

15. Tourneur, E. et al. Cyclosporine A impairs nucleotide binding oligomerization domain (Nod1)-mediated innate antibacterial renal defenses in mice and human transplant recipients. PLoS Pathog. 9, e1003152 (2013).

16. Kang, Y.J. et al. Calcineurin negatively regulates TLR-mediated activation pathways. J. Immunol. 179, 4598-4607 (2007).

17. Fric, J. et al. Calcineurin/NFAT signalling inhibits myeloid haematopoiesis. EMBO Mol. Med. 4, 269-282 (2012).

18. Fric, J., Zelante, T. \& Ricciardi-Castagnoli, P. Phagocytosis of particulate antigens - all roads lead to calcineurin/NFAT signaling pathway. Front. Immunol. 4, 513 (2014).

19. Fric, J. et al. Calcium and calcineurin-NFAT signaling regulate granulocytemonocyte progenitor cell cycle via Flt3-L. Stem Cells 32, 3232-3244 (2014).

20. Hermann, S. et al. Older patients with high-risk fungal infections can be successfully allografted using non-myeloablative conditioning in combination with intensified supportive care regimens. $\mathrm{Br}$. J. Haematol. 113, 446-454 (2001).

21. Zelante, T., Fric, J., Wong, A.Y. \& Ricciardi-Castagnoli, P. Interleukin-2 production by dendritic cells and its immuno-regulatory functions. Front. Immunol. 3, 161 (2012).

22. Zelante, T. et al. CD103(+) dendritic cells control Th17 cell function in the lung. Cell Rep. 12, 1789-1801 (2015).

23. Goodridge, H.S., Simmons, R.M. \& Underhill, D.M. Dectin-1 stimulation by Candida albicans yeast or zymosan triggers NFAT activation in macrophages and dendritic cells. J. Immunol. 178, 31073115 (2007).

24. Garlanda, C. et al. Non-redundant role of the long pentraxin PTX3 in antifungal innate immune response. Nature 420, 182-186 (2002).

25. Bottazzi, B. et al. The long pentraxin PTX3 as a prototypic humoral pattern recognition receptor: interplay with cellular innate immunity. Immunol. Rev. 227, 9-18 (2009).

26. Balhara, J., Koussih, L., Zhang, J. \& Gounni, A.S. Pentraxin 3: an immunoregulator in the lungs. Front. Immunol. 4, 127 (2013).

27. Bozza, S. et al. Pentraxin 3 protects from MCMV infection and reactivation through TLR sensing pathways leading to IRF3 activation. Blood 108, 3387-3396 (2006).
28. Deban, L., Jaillon, S., Garlanda, C., Bottazzi, B. \& Mantovani, A. Pentraxins in innate immunity: lessons from PTX3. Cell Tissue Res. 343, 237-249 (2011).

29. Branzk, N. et al. Neutrophils sense microbe size and selectively release neutrophil extracellular traps in response to large pathogens. Nat. Immunol. 15, 1017-1025 (2014).

30. Moalli, F. et al. Role of complement and Fc\{gamma\} receptors in the protective activity of the long pentraxin PTX3 against Aspergillus fumigatus. Blood 116, 5170-5180 (2010).

31. Cunha, C. et al. Genetic PTX3 deficiency and aspergillosis in stem-cell transplantation. N. Engl. J. Med. 370, 421-432 (2014).

32. Wojtowicz, A. \& Bochud, P.Y. Host genetics of invasive Aspergillus and Candida infections. Semin. Immunopathol. 37, 173-186 (2015).

33. Caton, M.L., Smith-Raska, M.R. \& Reizis, B. Notch-RBP-J signaling controls the homeostasis of CD8- dendritic cells in the spleen. J. Exp. Med. 204, 1653-1664 (2007).

34. Zeng, H. et al. Forebrain-specific calcineurin knockout selectively impairs bidirectional synaptic plasticity and working/episodic-like memory. Cell 107, 617-629 (2001).

35. Clausen, B.E., Burkhardt, C., Reith, W., Renkawitz, R. \& Forster, I. Conditional gene targeting in macrophages and granulocytes using LysMcre mice. Transgenic Res. 8, 265-277 (1999).

36. Baddley, J.W. et al. Factors associated with mortality in transplant patients with invasive aspergillosis. Clin. Infect. Dis. 50, 1559-1567 (2010).

37. Becker, K. L., Ifrim, D.C., Quintin, J., Netea, M.G. \& van de Veerdonk, F.L Antifungal innate immunity: recognition and inflammatory networks. Semin. Immunopathol. 37, 107-116 (2015).

38. Jaillon, S. et al. The humoral pattern recognition molecule PTX3 is a key component of innate immunity against urinary tract infection. Immunity 40 , 621-632 (2014).

39. Bozza, S. et al. PTX3 binds MD-2 and promotes TRIF-dependent immune protection in Aspergillosis. J. Immunol. 193, 2340-2348 (2014).

40. Altmeyer, A., Klampfer, L., Goodman, A.R. \& Vilcek, J. Promoter structure and transcriptional activation of the murine TSG-14 gene encoding a tumor necrosis factor/interleukin-1-inducible pentraxin protein. J. Biol. Chem. 270, 25584-25590 (1995).

41. Jaillon, S. et al. The humoral pattern recognition receptor PTX3 is stored in neutrophil granules and localizes in extracellular traps. J. Exp. Med. 204, 793-804 (2007).

42. Gaziano, R. et al. Anti-Aspergillus fumigatus efficacy of pentraxin 3 alone and in combination with antifungals. Antimicrob. Agents Chemother. 48, 4414-4421 (2004).

43. Lo Giudice, P. et al. Efficacy of PTX3 in a rat model of invasive aspergillosis. Antimicrob. Agents Chemother. 54, 4513-4515 (2010).

44. Bochud, P.Y. et al. Toll-like receptor 4 polymorphisms and aspergillosis in stem-cell transplantation. N. Engl. J. Med. 359, 1766-1777 (2008).

45. Carvalho, A. et al. Polymorphisms in toll-like receptor genes and susceptibility to pulmonary aspergillosis. J. Infect. Dis. 197, 618-621 (2008).

46. Sainz, J. et al. Dectin-1 and DC-SIGN polymorphisms associated with invasive pulmonary Aspergillosis infection. PLoS One 7, e32273 (2012).

47. Cunha, C. et al. Dectin-1 Y238X polymorphism associates with susceptibility to invasive aspergillosis in hematopoietic transplantation through impairment of both recipient- and donor-dependent mechanisms of antifungal immunity. Blood 116, 5394-5402 (2010).

48. Chai, L.Y. et al. The Y238X stop codon polymorphism in the human betaglucan receptor dectin-1 and susceptibility to invasive aspergillosis. J. Infect. Dis. 203, 736-743 (2011).

49. Kuhn, R., Schwenk, F., Aguet, M. \& Rajewsky, K. Inducible gene targeting in mice. Science 269, 1427-1429 (1995).

50. Bozza, S. et al. Dendritic cells transport conidia and hyphae of Aspergillus fumigatus from the airways to the draining lymph nodes and initiate disparate Th responses to the fungus. J. Immunol. 168, 1362-1371 (2002). 